\title{
Maturation of human pluripotent stem cell derived cardiomyocytes is improved in cardiovascular construct
}

\author{
Hanna Vuorenpää • Kirsi Penttinen (1) - Tuula Heinonen • Mari Pekkanen-Mattila • \\ Jertta-Riina Sarkanen • Timo Ylikomi • Katriina Aalto-Setälä
}

Received: 4 November 2016/Accepted: 17 March 2017/Published online: 10 April 2017

(C) The Author(s) 2017. This article is an open access publication

\begin{abstract}
In order to translate preclinical data into the clinical studies, relevant in vitro models with structure and key functional properties similar to native human tissue should be used. In vitro cardiac models with vascular structures mimic the highly vascularized myocardium and provide interactions between endothelial cells, stromal cells and cardiomyocytes. Currently, human pluripotent stem cellderived cardiomyocytes (hPSC-CMs) have been shown to present immature morphology and fetal-like
\end{abstract}

Electronic supplementary material The online version of this article (doi:10.1007/s10616-017-0088-1) contains supplementary material, which is available to authorized users.

Hanna Vuorenpää and Kirsi Penttinen have contributed equally to this work.

H. Vuorenpää · T. Heinonen · T. Ylikomi

FICAM, Finnish Centre for Alternative Methods, Faculty

of Medicine and Life Sciences, University of Tampere,

PL 100, 33014 Tampere, Finland

e-mail: hanna.k.vuorenpaa@gmail.com

T. Heinonen

e-mail: tuula.heinonen@staff.uta.fi

T. Ylikomi

e-mail: timo.ylikomi@staff.uta.fi

K. Penttinen · M. Pekkanen-Mattila · K. Aalto-Setälä BioMediTech, Institute of Biomedical Technology, Faculty of Medicine and Life Sciences, University of Tampere, PL 100, 33014 Tampere, Finland

e-mail: mari.pekkanen-mattila@uta.fi electrophysiological properties that may limit their use as physiological test platform. The aim of this study was to develop multicellular in vitro cardiovascular construct modeling human heart tissue. In the cardiovascular construct, hPSC-CMs were cultured with a vascular-like network formed by human foreskin fibroblasts and human umbilical vein endothelial cells that served as a platform in the construct. Cardiomyocyte orientation, maturation, electrophysiological properties and drug responses of the cardiovascular construct were characterized and compared to $\mathrm{CM}$ monoculture. hPSC-CMs in cardiovascular construct showed elongated morphology and aligned with the vascular-like network. Electrophysiological properties and calcium metabolism of hPSC-CMs as well as

K. Penttinen $(\bowtie) \cdot T$. Ylikomi · K. Aalto-Setälä Faculty of Medicine and Life Sciences, University of Tampere, PL 100, 33014 Tampere, Finland e-mail: kirsi.penttinen@uta.fi

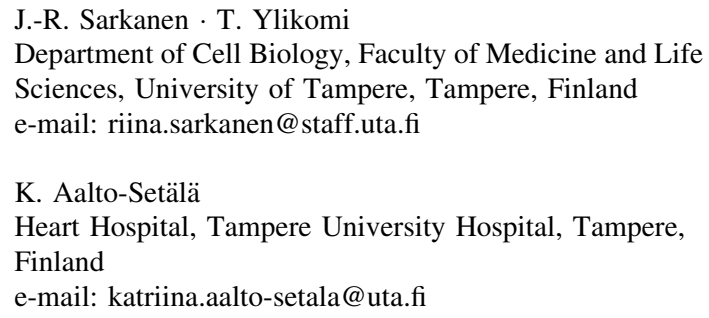


response to E-4031 and adrenaline demonstrated normal physiological behavior. Increased expression of cardiac structural proteins and ion channels in cardiovascular construct compared to $\mathrm{CM}$ monoculture were detected. In conclusion, vascular-like network supports the structural and functional maturation of hPSC-CMs. Our results suggest that cardiovascular construct presents more mature in vitro cardiac model compared to $\mathrm{CM}$ monoculture and could therefore serve as an advanced test system for cardiac safety and efficacy assessment as well as a model system for biomedical research.

Keywords Cardiomyocytes - Vascular-like network · Maturation

\section{Introduction}

In adult heart, the majority of cardiac cells are highly adaptive cells including fibroblasts, vascular smooth muscle cells and endothelial cells. 20-40\% of the cardiac cells are cardiomyocytes (Soonpaa and Field 1998; Brutsaert 2003). In cardiac microenvironment, cardiomyocytes are embedded in aligned extracellular matrix (ECM) that facilitates the coordinated contractile function of the heart (Chien et al. 2008). Cells and ECM proteins, mainly produced by fibroblasts, are connected via cell-cell and cellmatrix interactions to maintain the structural organization and functionality of the heart (van Spreeuwel et al. 2014; Pfannkuche et al. 2010b). In mature myocardium, each cardiomyocyte has physical contact with at least one capillary blood vessel (Garzoni et al. 2009). The interactions between vasculature and myocardium are bidirectional (Bhattacharya et al. 2006), and active throughout the adult life affecting cardiac growth, function and rhythm (Brutsaert 2003). From the onset of the cardiac development, endothelial cells are the prerequisite for myocardial maturation, physiological function and survival (Brutsaert 2003). Endothelial cells and cardiomyocytes interact with each other with paracrine signals during heart development and activate the development of cardiac structure. This interaction is required for proper development of endocardium and myocardium. Vascular endothelial cells produce several compounds including angiopoietin 2
(Ang-2), whereas angiopoietin 1 (Ang-1) and vascular endothelial growth factor (VEGF) are mainly produced by cardiomyocytes (Brutsaert 2003; Leucker et al. 2011; Hsieh et al. 2006). In addition, fibroblasts secrete factors with autocrine and paracrine effects, such as VEGF, fibroblast growth factors (FGFs) and transforming growth factor beta (TGF- $\beta$ ), that give mechanical support and promote the organization of cardiomyocytes into 3D structures in collagen matrices (Pfannkuche et al. 2010b; Wong et al. 2007).

Cardiotoxicity is one of the leading causes of failure for a new therapeutic molecule preclinical development (Braam et al. 2010). Presently, pharmaceutical industry relies upon animal testing, although there are fundamental differences in the electrophysiological properties of animal and human cardiomyocytes (Feric and Radisic 2016). In particular, the differences in ion channels and currents impact the poor predictivity of drug screening and toxicity studies from murine to humans (Polini et al. 2014). In addition to animal models, transfected non-cardiac cells have been used in preclinical testing. However, these cells express usually only one cardiac ion channel and lack other characteristics of human cardiomyocytes. Transfected non-cardiac cells lack a cardiac intracellular environment that includes cardiomyocyte specific accessory proteins, as well as, cell structure (Martin et al. 2004). Therefore, there is an unmet need for improved human cardiomyocyte model for preclinical drug screening especially for assessment of cardiotoxic effects and for evaluation of the efficacy of new drug candidates (Feric and Radisic 2016; Kettenhofen and Bohlen 2008). The cell model should mimic effectively human cardiomyocytes for example in terms of cell structure, cardiac specific ion channels and electromechanical function.

Functional cardiomyocytes derived from human pluripotent stem cells could provide a significant advantage over the previously used test systems. Human pluripotent stem cell derived cardiomyocytes (hPSC-CMs) contract spontaneously and respond appropriately to cardioactive drugs (Robertson et al. 2013). These cells could be useful in early efficacy and toxicity screening, improving the selection of lead candidates and the reduction of adverse outcomes in clinical stages of drug development (Davila et al. 2004). According to their morphological and functional characteristics, hPSC-CM have been shown to 
display properties typical to human fetal cardiomyocytes which may complicate their utilization and interpretation of the obtained results (Snir et al. 2003; Robertson et al. 2013). Compared to the human adult cardiomyocytes the hPSC-CMs have been reported to have lower expression of the genes related to the ion transportation, calcium handling and sarcomere structure (Yang et al. 2014). Furthermore, the morphology of the hPSC-CMs does not resemble the well-aligned rod-like nature of adult human cardiomyocytes. Therefore, the maturation level of hPSC-CMs can be assessed by measuring the expression levels of genes encoding sarcomeric proteins such as cardiac Troponin $I$ and $T$ as well as $\alpha$ - and $\beta$ myosin heavy chains. In addition, the expression levels of the cardiac specific ion channels and connexin 43 responsible for the intracellular communication can be used in estimation of the maturation state (Sartiani et al. 2007). In addition to the increased time in culture, electrical stimulation (Chan et al. 2013) and 3D culture environment (Schaaf et al. 2011; van Spreeuwel et al. 2014; Garzoni et al. 2009; Valarmathi et al. 2010; Pontes Soares et al. 2012) have been utilized in the production of more mature hPSC-CMs. However, the use of scaffolds to enable 3D environment has been associated with reduced cell-cell contacts, as well as incorrect deposition and alignment of extracellular matrix in cardiac constructs (Norotte et al. 2009).

In this study, a multicellular in vitro cardiovascular construct was developed. In the construct, hPSC-CMs were cultured with vascular-like network composed of human fibroblasts and human umbilical vein endothelial cells (HUVEC). Vascular-like network serves as a supporting platform to create natural microenvironment, i.e. biological scaffold, with cell-cell and cellmatrix interactions. The effects of the vascular-like network on cardiomyocyte morphology, gene expression and functionality were studied. Results demonstrated that the vascular-like network enhanced the structural maturation and increased the expression levels of cardiac ion channels in hPSC-CMs in the cardiovascular construct. Our study suggests that this cardiovascular construct provides a more mature in vitro cardiac model compared to $\mathrm{CM}$ monoculture and could therefore serve as an advanced test system for cardiac safety and efficacy assessment as well as a model system in biomedical research.

\section{Materials and methods}

Ethics

This study conforms to the principles outlined in the Declaration of Helsinki. The use of human umbilical vein endothelial cells (HUVEC) and induced pluripotent stem cells (iPSC) were approved by Ethics Committee of the Pirkanmaa Hospital District, Tampere, Finland (Approval Numbers R08028 and R08070, respectively) and a written informed consent was obtained from all the participants.

Cell culture and differentiation

Isolation and culture of human umbilical vein endothelial cells

HUVECs were isolated from human umbilical cord vein by using enzymatic procedure as described previously by us (Sarkanen et al. 2011). Cells were detached from umbilical cords with $0.05 \%$ collagenase I, cultured in EGM-2 medium (Lonza Group Ltd, Basel, Switzerland, Table 1) and tested for mycoplasma contamination (MycoAlert ${ }^{\circledR}$ Mycoplasma Detection Kit, Lonza Group Ltd) before experimental use. We use HUVEC as a standard source of endothelial cells in cell culture studies in our laboratory. The isolation and culture process of HUVECs has been established and validated producing cell lines with low variability (Sarkanen et al. 2011). Cells are routinely stored in liquid nitrogen in passage 2 and used for establishing cell culture models in passage 4 . Each HUVEC line is derived from a single donor and quality tested routinely for their ability to form tubule structures in cell culture.

\section{Culture of human foreskin fibroblasts}

Human foreskin fibroblasts were purchased from American Type Culture Collection (BJ, CRL-2522; ATCC, Manassas, VA, USA). Cells were cultured in fibroblast medium (Table 1) consisting of Minimum Essential Medium with Earle's salts, w/o L-glutamine (Gibco, Vantaa, Finland) supplemented with 10\% FBS (Gibco), 1\% L-glutamine (Gibco) and 1\% NEAA (Gibco). Cells were tested for mycoplasma 
Table 1 Culture media used for development of cell models

\begin{tabular}{lllll}
\hline Acronym & $\begin{array}{l}\text { Basal } \\
\text { medium }\end{array}$ & Serum & Growth factors & Supplementation \\
\hline Fibroblast medium & MEM & $10 \%$ FBS & - & $1 \%$ L-glutamine, 1\% NEAA \\
EGM-2 medium & EBM-2 & $2 \%$ FBS & $\begin{array}{c}\text { VEGF, FGF-2, } \\
\text { IGF, EGF }\end{array}$ & $\begin{array}{c}\text { Hydrocortisone, ascorbic } \\
\text { acid, heparin }\end{array}$ \\
Angiogenic stimulation medium & EBM-2 & $2 \%$ FBS & 10 ng/ml VEGF, & $1 \%$ L-glutamine \\
EB 5\% & DMEM/F12 & $5 \%$ FBS & - & $1 \%$ NEAA, \\
& & & & \\
& & & & $0.5 \%$ Pen/Strep \\
\hline
\end{tabular}

$V E G F$ vascular endothelial growth factor, $F G F-2$ fibroblast growth factor 2, EGF epidermal growth factor

contamination (MycoAlert ${ }^{\circledR}$ Mycoplasma Detection Kit, Lonza) before experimental use.

\section{Generation of patient-specific iPSC line and cell culture of pluripotent stem cells}

In this study one commercial human embryonic stem cell (hESC) line H7 purchased from WiCell Research Institute (Madison, WI, USA) and one iPSC line was used for cardiomyocyte differentiation. Patient-specific iPSC line UTA.04602. WT was established from a healthy individual as described earlier (Takahashi et al. 2007). Shortly, skin biopsy from the donor was cultured in $0.2 \%$ gelatin (Sigma-Aldrich, Espoo, Finland) coated flask under fibroblast culturing conditions. iPSC line was established using lentivirus infection followed by retrovirus infection (Takahashi et al. 2007). Cells, plasmids and reagents used in this protocol include: 293FT cells, Plat-E cells, pLenti6/ UbC/mSlc7a1-vector (Addgene, Cambridge, MA, USA), ViraPower ${ }^{\mathrm{TM}}$ Packaging Mix (Life Technologies Ltd), Lipofectamine ${ }^{\mathrm{TM}} 2000$ (Life Technologies Ltd, Vantaa, Finland), Fugene 6 (Roche Diagnostics, Mannheim, Germany), and pMX retroviral vectors (hOCT3/4, hSOX2, hKLF4 and hc-MYC, all from Addgene). Results of the characterization of UTA.04602.WT cell line have been described earlier (Lahti et al. 2012).

UTA.04602.WT cells and $\mathrm{H} 7 \mathrm{hESCs}$ were cultured on mitomycin $\mathrm{C}$ inactivated mouse embryonic fibroblasts (MEF) in KSR medium which consisted of DMEM/F-12 (Invitrogen) supplemented with 20\% KnockOut serum replacement (Invitrogen), 1\% nonessential amino acids (Lonza), $2 \mathrm{mM}$ Glutamax (Invitrogen), $50 \mathrm{U} / \mathrm{ml}$ penicillin/streptomycin
(Lonza), $0.1 \mathrm{mM}$ beta mercaptoethanol (Invitrogen) and $7.8 \mathrm{ng} / \mathrm{ml}$ basic fibroblast growth factor (R\&D Systems). The medium was refreshed daily, and the stem cell colonies were passaged onto a new MEF layer once a week using $1 \mathrm{mg} / \mathrm{ml}$ collagenase IV (Invitrogen).

\section{Differentiation of cardiomyocytes}

Differentiation of pluripotent stem cells into cardiomyocytes was carried out with either by coculturing hESC or iPSC with murine visceral endoderm-like (END-2) cells (Humbrecht Institute, Utrecht, The Netherlands) as described earlier (Mummery et al. 2003) or with small molecule differentiation method via temporal modulation of canonical Wnt signaling (Lian et al. 2013). Briefly, in END-2 method undifferentiated hPSC colonies were dissected mechanically into aggregates and plated on top of mitomycin C (Tocris) treated END-2 cells in the hPSC medium. The culture medium was supplemented with $2.92 \mathrm{mg} / \mathrm{ml}$ of ascorbic acid (Sigma-Aldrich). The medium was refreshed on days 5, 8 and 12. On day 14, $10 \%$ serum replacement was added to the medium. Briefly, for small molecule differentiation hPSCs maintained on a Geltrex-coated surface in mTeSR1 (Stemcell Technologies, Cologne, Germany) were dissociated into single cells with Accutase (Life Technologies) at $37^{\circ} \mathrm{C}$ for $5 \mathrm{~min}$ and then seeded onto a Geltrex-coated cell culture dish at 50,000 cell/ $\mathrm{cm}^{2}$ in mTeSR1 supplemented with $5 \mu \mathrm{M}$ ROCK inhibitor (Tocris, Minneapolis, MN, USA) for $24 \mathrm{~h}$. Cells were then cultured in mTeSR1, changed daily. On differentiation day 0 , medium was exchanged with RPMI medium (Life Technologies) with $1 \times \mathrm{B} 27^{\circledR}$ 
Supplement minus insulin (Life Technologies) with $10 \mu \mathrm{M}$ CHIR99021 (Stemgent, Lexington, MA, USA). $24 \mathrm{~h}$ later, medium was exchanged with RPMI/B27 without insulin. On day 3, medium was exchanged with RPMI/B27 without insulin supplemented with $5 \mu \mathrm{M}$ IWP4 (Miltenyi Biotech, Bergisch Gladbach, Germany). On day 5, medium was exchanged with RPMI/B27 without insulin. On day 7 and every 3 days following, medium was exchanged with RPMI/B27.

Both differentiation methods formed beating cardiomyocyte aggregates. These were mechanically excised and treated with collagenase A (Roche Diagnostics) to dissociate beating aggregates to single cell level (Mummery et al. 2003). In each experiment, cardiomyocytes plated on the monoculture and on top of the vascular-like network originated from the same differentiation and dissociation batch.

Development of cell models

\section{Human cardiomyocyte monoculture}

After dissociation, human hPSC-CMs were seeded in EB 5\% medium (Table 1) at density of $0.01-0.04 \times 10^{6}$ cells $/ \mathrm{cm}^{2}$ in $0.1 \%$ gelatin type A (Sigma-Aldrich) coated 48-well plates for immunocytochemical and qRT-PCR analyses and in microelectrode array (MEA) platforms and gelatin coated $12 \mathrm{~mm}$ cover slips for functional analyses. EB 5\% medium was changed 1-2 days after cell seeding and thereafter twice a week. hPSC-CM monoculture in EB $5 \%$ medium was used as a control throughout the study.

Fig. 1 Establishment of cardiovascular construct
Vascular-like network from co-culture of HUVEC and fibroblast

The co-culture was established as described earlier (Sarkanen et al. 2011). Briefly, fibroblasts (p 6-7), were seeded at 20,000 cells $/ \mathrm{cm}^{2}$ in fibroblast medium (Table 1) in 48-well plates for immunocytochemical and qRT-PCR analyses and in MEA-platforms and $12 \mathrm{~mm}$ diameter cover slips for functional analyses and grown for 2-3 days to confluency. HUVEC were seeded on top of confluent fibroblast cultures at 4000 cells $/ \mathrm{cm}^{2}$ in EGM-2 medium (Table 1). The day after cell seeding, angiogenic stimulation medium containing EBM-2 (Lonza), 2\% FBS, $1 \mathrm{mM}$ L-glutamine, $10 \mathrm{ng} / \mathrm{ml}$ vascular endothelial growth factor (VEGF, Sigma) and $1 \mathrm{ng} / \mathrm{ml}$ fibroblast growth factor 2 (FGF-2, Sigma) (Table 1) was applied to cells. The angiogenic stimulation medium was changed twice during the 6 day co-culture prior to CM seeding.

\section{Cardiovascular construct}

EB 5\% medium (Table 1) was changed to HUVEC + fibroblast co-culture before seeding hPSC-CMs. Dissociated hiPSC- or hESC-derived cardiomyocytes in EB 5\% medium (Fig. 1) were seeded on top of the HUVEC + fibroblast co-culture at day 6 , when the vascular-like network was already formed, at a density of $0.01-0.04 \times 10^{6}$ cells $/ \mathrm{cm}^{2}$ in 48-well plates for immunocytochemical and qRTPCR analyses and in microelectrode array (MEA) platforms and $12 \mathrm{~mm}$ diameter cover slips for functional analyses. 1-2 days after cardiomyocyte seeding first EB medium change was performed and thereafter
Seeding of cardiomyocytes (removal of VEGF and FGF-2 prior to seeding)

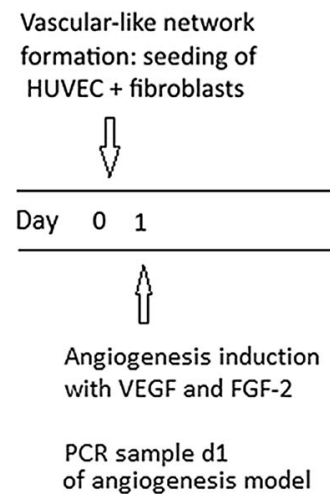

PCR sample $d 6$ of angiogenesis mode

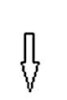

MEA measurements PCR sample d18 of angiogenesis model and cardiovascular construct Ca imaging, MEA measurements

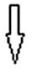

\begin{tabular}{llll}
67 & 10 & 18 \\
\hline
\end{tabular}

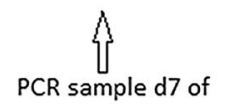

cardiovascular construct 
three times in a week. The viability of cardiovascular constructs was evaluated visually under microscope by assessing the contraction of the cardiomyocytes and vascular-like network formation of the HUVECs and fibroblasts at least three times in a week.

Medium development

In cardiovascular construct, medium was designed to primarily support cardiomyocyte functionality and secondarily to induce vascular-like network formation. EB medium (Table 1) with different serum concentrations including 0,2, 5, 10, and 20\% FBS (Biosera-Immuno Diagnostic Oy, Hämeenlinna, Finland) were tested with duplicate wells for dissociated cardiomyocytes due to our objective to have lowserum or serum-free culture conditions, to avoid exogenous bias in our cell culture system and to develop in vitro model that mimics closely the in vivo situation in order to improve the predictive value of the test system. In that, it is important to provide physiologically relevant environment with low/noserum culture conditions. Cells were cultured for 9 days and fixed with 4\% PFA (Sigma-Aldrich) for immunocytochemical stainings with goat-anti-cardiac troponin-T (anti-Tnt, 1:1500, Abcam, Cambridge, U.K.). Cells were mounted with Vectashiel (Vector Laboratories, Burlingame, CA, USA) containing DAPI for staining nuclei. The results of the serum concentration tests can be found in the supplemental data (Fig. S1).

\section{Immunocytochemistry}

Cardiomyocytes in CM monoculture and in cardiovascular construct were stained either with goat anticardiac-troponin-T (anti-Tnt, 1:1500, Abcam) or with mouse anti-Tnt (1:500, Abcam) and the vascular-like formation was visualized with basement membrane marker mouse collagen IV (anti-ColIV, 1:500, Sigma) for $1 \mathrm{~h}$ at $\mathrm{RT}$ or in $+4{ }^{\circ} \mathrm{C}$ o/n. Polyclonal IgG Alexa Fluor 568 (Abcam) for goat anti-Tnt and polyclonal IgG FITC (1:100, Sigma) for anti-ColIV and mouse anti-Tnt were used as secondary antibodies for $30 \mathrm{~min}$ in RT. Fluorescence was visualized with Nikon Eclipse Ti-S microscope (Nikon, Otawara, Tochigi, Japan) or with confocal laser scanning microscope Zeiss LSM780 Laser Scanning Confocal Microscope (ZEISS, Oberkochen, Germany). The images were processed with Zen2009 (confocal images, Carl Zeiss) and with Adobe Photoshop software 7.0 (Adobe Systems). The results are from at least 4 individual experiments performed in duplicates.

Quantitative real time-PCR

Gene expression in CM monoculture, vascular-like network and cardiovascular construct were analyzed. The total RNA was extracted at day 1, 6, 7 and 18 (Fig. 1) using PureLink RNA Mini Kit (Life Technologies) following the manufacturer's protocol. Concentration and purity of RNA was assessed using spectrophotometry with microplate reader in Varioskan Flash Spectrophotometer (ThermoScientific, Espoo, Finland) before further use. Reverse transcription of the total RNA to cDNA was performed using iScript cDNA synthesis kit (Bio-Rad, Helsinki, Finland) following manufacturer's instructions. qRTPCR was performed according to standard protocols on Abi Prism 7300 instrument (Applied Biosystems, Espoo, Finland) or on Bio-rad CFX96 Real Time System (BioRad). For each time point two biological replicate samples were collected and samples were analyzed as triplicates. The expression of angiogenesis related genes including VEGF-A, FGF-2, PDGF- $\beta$, TGF- $\beta 1$, Angiopoietin-1, Angiopoietin-2 and cardiac related genes including CACNA1C, TNNT2, KCNJ2, CX43, MYH6, MYH7 were studied with SYBR chemistry. In addition, the expression of cardiac related genes $A D R B 1$ and $S C N 5 a$ as well as reference gene $G A P D H$ were studied using Taqman chemistry with Taqman Universal PCR Master Mix (Applied Biosystems). The following Taqman assays $(20 \times)$ were used: Hs02330048_s1 for ADRB1, Hs00165693_m1 for SCN5A and Hs02758991_g1 for GAPDH (Applied Biosystems). SYBR primer sequences can be found in supplemental data (Supplementary Table 1). The relative expression levels were determined by using the comparative method $(\Delta \Delta \mathrm{Ct})$ and GAPDH was used as an endogenous control (Livak and Schmittgen 2001, 402-408).

Functional analyses

\section{Microelectrode array (MEA) measurements}

The ability of cardiovascular construct to conduct electrical signal was analyzed using the MEA system 
(Multi Channel Systems MCS GmbH, Reutlingen, Germany). The MEA platforms $(8 \times 8$ standard MEAs or 6-well MEAs) were first hydrophilized with FBS and then coated with $0.1 \%$ gelatin type A (SigmaAldrich).

Field potentials were recorded at day 10 or 18 (Fig. 1) at $37{ }^{\circ} \mathrm{C}$, and signals were recorded for $2 \mathrm{~min}$. The sampling frequency was $20 \mathrm{kHz}$. Field potentials were recorded during spontaneous baseline beating, and with $1 \mu \mathrm{M}$ adrenaline (Sigma-Aldrich) or $300 \mathrm{nM}$ E-4031 (Sigma-Aldrich), which were incubated $2 \mathrm{~min}$ before measurements. Drugs were diluted and measurements performed in EB 5\% medium. The field potentials were recorded with MC_Rack v.4.5.7 software (Multi Channel Systems MCS GmbH). Signals were analyzed with Cardiomyocyte MEA Data Analysis (CardioMDA) software (Pradhapan et al. 2013).

\section{Analysis of Calcium $^{2+}$ cycling}

Cardiovascular constructs were loaded with $4 \mu \mathrm{mol} / \mathrm{L}$ Fura-2 AM (Invitrogen, Molecular Probes) for $30 \mathrm{~min}$ in HEPES based medium. Measurements were assessed in $37^{\circ} \mathrm{C}$ and the extracellular solution consisted of (in mmol/L): $137 \mathrm{NaCl}, 5 \mathrm{KCl}, 0.44$ $\mathrm{KH}_{2} \mathrm{PO}_{4}, 20$ HEPES, $4.2 \mathrm{NaHCO}_{3}, 5$ D-glucose, 2 $\mathrm{CaCl}_{2}, 1.2 \mathrm{MgCl}_{2}$ and $1 \mathrm{Na}$-pyruvate ( $\mathrm{pH}$ was adjusted to 7.4 with $\mathrm{NaOH}$ ).

$\mathrm{Ca}^{2+}$ measurements were recorded at day 10 or 11 (Fig. 1) and conducted on an inverted IX70 microscope (Olympus Corporation, Vantaa, Finland) with a UApo/340 20× air objective (Olympus). Images were acquired with an ANDOR iXon 885 CCD camera (Andor Technology, Belfast, U.K.) synchronized with a Polychrome V light source by a real time DSP control unit and TILLvisION software (TILL Photonics, Gräfelfing, Germany). Fura-2 in cardiomyocytes was excited at 340 and $380 \mathrm{~nm}$ light and the emission was recorded at $505 \mathrm{~nm}$. For $\mathrm{Ca}^{2+}$ analysis, regions of interests were selected for spontaneously beating cells and background noise was subtracted before further processing. The $\mathrm{Ca}^{2+}$ levels are presented as ratiometric values of F340/F380. The changes in $\mathrm{Ca}^{2+}$ were recorded during spontaneous baseline beating and spontaneous beating after 2-min $1 \mu \mathrm{M}$ adrenaline (Sigma-Aldrich) perfusion.
Statistics

qPCR data are expressed as mean values \pm SD. Statistical analysis for the qPCR data was performed by IBM SPPS Statistics 22-software and by using Mann-Whitney $U$-test for independent samples. Bonferroni-correction was included when more than two groups were analyzed. A $p$ value less than 0.05 was considered statistically significant.

In MEA and $\mathrm{Ca}^{2+}$ imaging data, the significance of differences between the two groups (CM monoculture and cardiovascular construct) was evaluated with the unpaired Student's $t$ test. The significance of changes within a group was evaluated with the paired Student's $t$ test. MEA and $\mathrm{Ca}^{2+}$ imaging data are expressed as average \pm SEM. In $\mathrm{Ca}^{2+}$ imaging data $\mathrm{n}$ refers to the number of cells and in MEA data $n$ refers to number of MEA wells. A $p$ value less than 0.05 was considered statistically significant.

\section{Results}

Vascular-like network serves as a supporting and interactive platform for cardiovascular construct

Expression levels of angiogenesis related genes were analyzed at days 1, 6 and 18 in vascular-like network to assess the production of growth factors and maturation state of vascular structures (Fig. 2). During the first six days the expression of fibroblast growth factor 2 (FGF-2), transforming growth factor $\beta$ (TGF$\beta$ ) and angiopoietin-1 (Ang-1) had an increasing trend whereas VEGF expression remained constant. Statistically significant increase was observed in angiopoietin 2 (Ang-2) and platelet derived growth factor $\beta$ $(P D G F-\beta)$ expression. During the days 6-18, the expression of aforementioned genes remained constant except for Ang-2, which had significantly increased expression level.

The expression levels of the angiogenesis related genes were also assessed from the cardiovascular construct at day 6 and 18 (Fig. 2). The expression levels of $F G F-2, T G F-\beta$ and angiopoietin-1 remained quite constant during culture, whereas Ang-2 expression was decreased in cardiovascular construct 

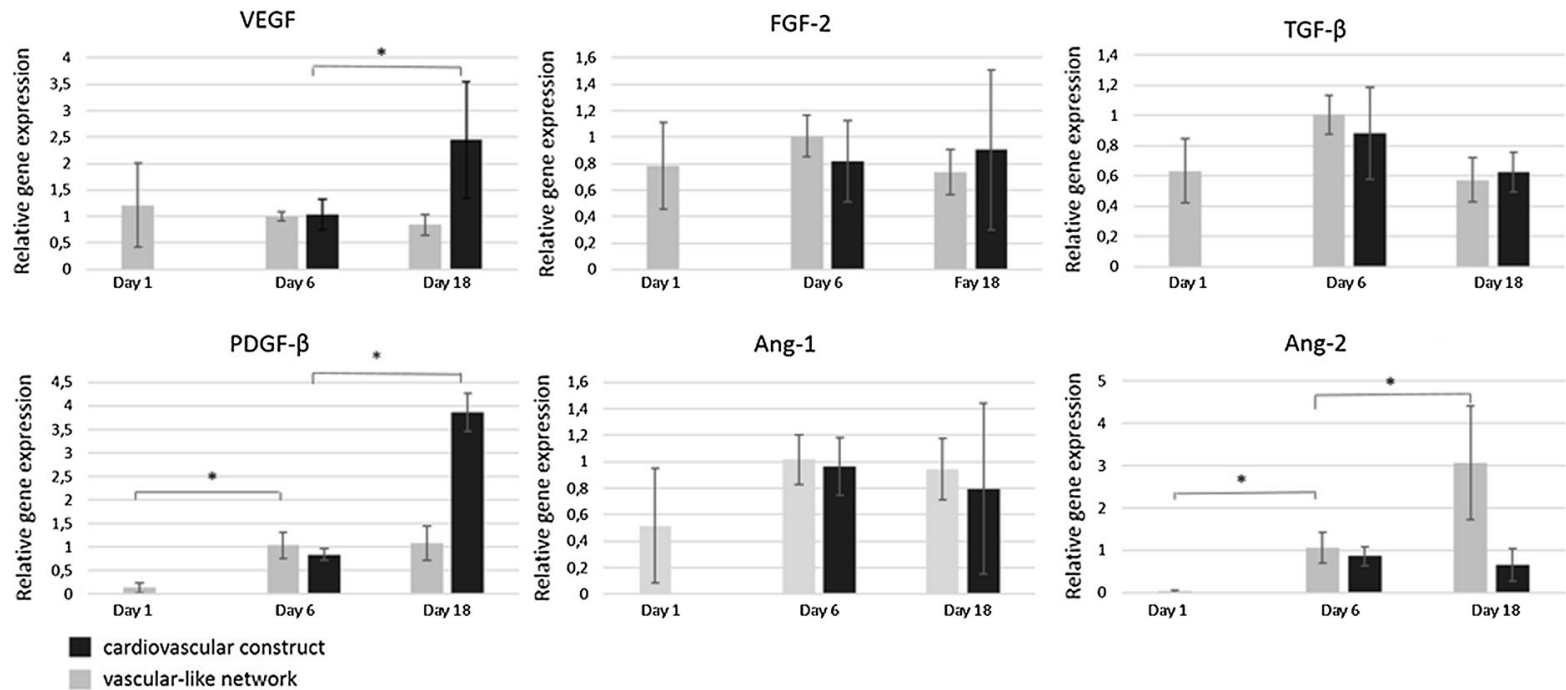

vascular-like network

Fig. 2 Relative gene expression levels of angiogenesis related genes including vascular endothelial growth factor (VEGF), fibroblast growth factor $2(F G F-2)$, transforming growth factor beta $(T G F-\beta)$, angiopoietin 1 (Ang-1), angiopoietin 2 (Ang-2) and platelet derived growth factor beta $(P D G F-\beta)$ in cardiovascular construct and in vascular-like network at time points 1,6 and 18. The GAPDH was used as a endogenous control. ${ }^{*} p<0.05$
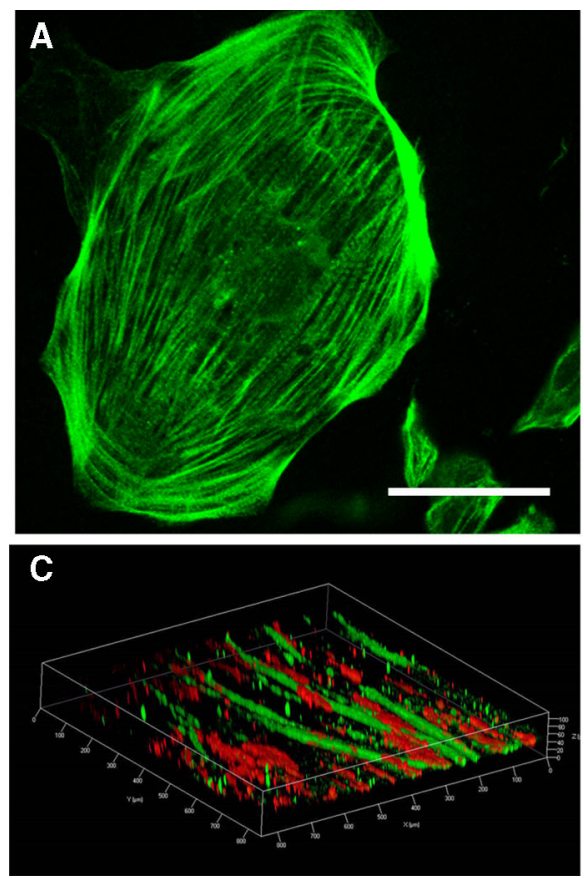

Fig. 3 Morphology of human pluripotent stem cell derived cardiomyocytes in $\mathbf{a}$ monoculture and in $\mathbf{b}$ cardiovascular construct at day 14 in $10 \%$ FBS. Orientation of human pluripotent stem cell derived cardiomyocytes (troponin $\mathrm{T}, \mathrm{red}$ ) with vascular-like network (collagen IV, green) in c,
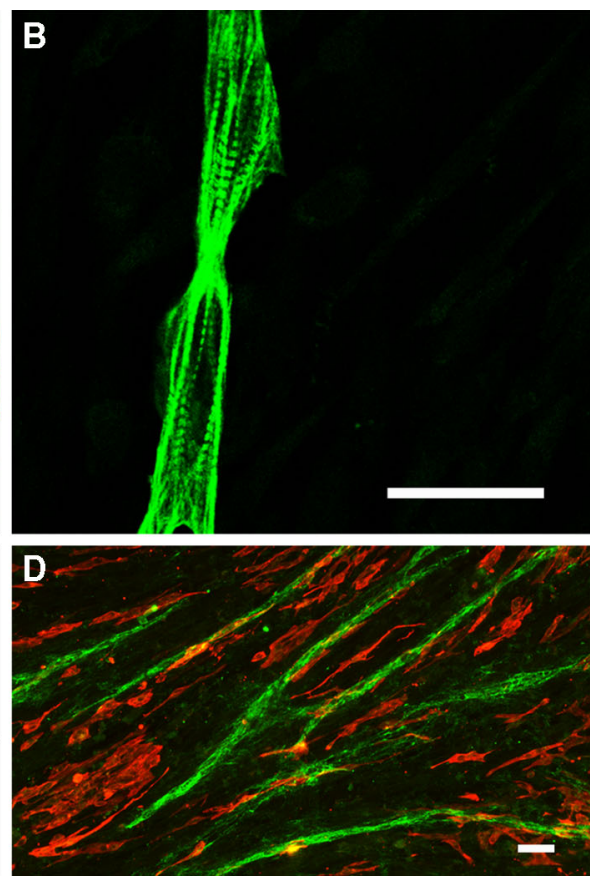

d cardiovascular construct at day 7 in 5\% FBS. C Confocal 3D z-stack projection having the scale bars included in the image. The vertical height is $100 \mu \mathrm{m}$ and the horizontal length between two major tick intervals is also $100 \mu \mathrm{m}$. In a, b and d the scale bars represent $50 \mu \mathrm{m}$. (Color figure online) 

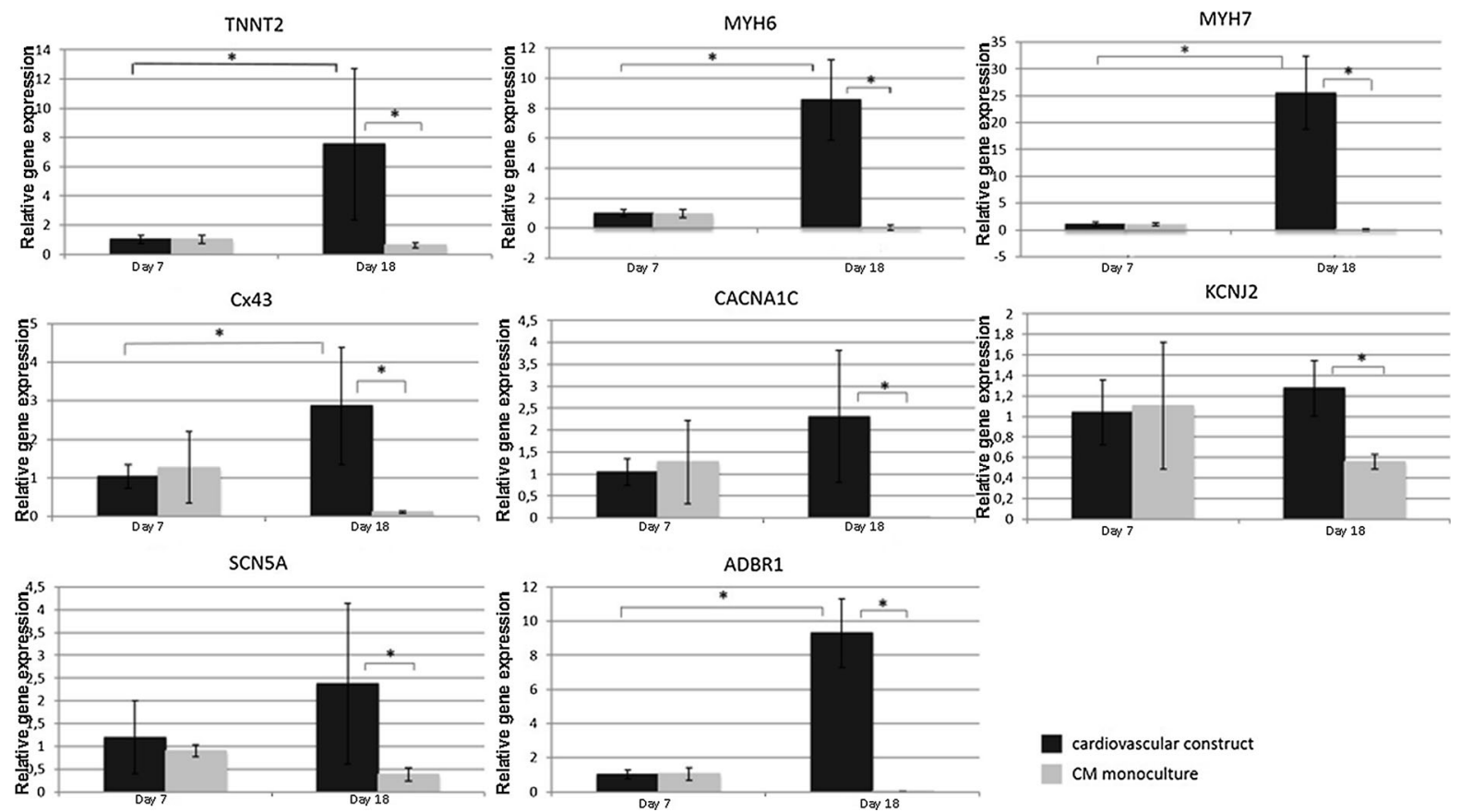

cardiovascular construct

$\mathrm{CM}$ monoculture

Fig. 4 Relative gene expression levels of cardiac related genes troponin T (TNNT2), cardiac muscle myosins (MYH6, MYH 7), connexin $43(C x 43)$, calcium channel (CACNA1C), potassium channel (KCNJ2), sodium channel (SCN5A) and beta-1- adrenergic receptor $(A D B R 1)$ at time points day 7 and day 18 in cardiomyocyte monoculture and in cardiovascular construct. The GAPDH was used as an endogenous control. * $p<0.05$ compared to the vascular-like network. However, the expression levels of $V E G F$ and $P D G F-\beta$ were significantly higher in the cardiovascular construct.

Vascular-like network enhances mature cardiac phenotype and orientation of pluripotent stem cell derived cardiomyocytes in cardiovascular construct

Compared to the round morphology and the random orientation of cardiomyocytes in monoculture (Fig. 3a; S1), more mature morphology with elongated cardiomyocytes was detected in the presence of vascular-like network (Fig. 3b). Moreover, 2D and 3D projections showed that cardiomyocytes co-localized longitudinally and parallel with tubular structures (Fig. 3c, d). On the contrary, cardiomyocytes in monoculture remained rounded, less organized and the orientation of the cells along the tubule structures could not be detected.

The expression levels of cardiac related genes were analyzed in the cardiovascular construct and in the CM monoculture at one day after cardiomyocyte seeding (day 7) and at day 18 (Fig. 4). Results showed that the expression of cardiac muscle myosin transcripts $M Y H 6$ and $M Y H 7$ as well as cardiac troponin $T$ increased between day 7 and 18 $(p<0.05)$ whereas the expression remained constant or decreased in the CM monoculture. The expression level of gap junction marker connexin 43 was also shown to increase significantly in cardiovascular construct $(p=0.002)$ between day 7-18 while remaining low in the $\mathrm{CM}$ monoculture. At day 18 the expression levels of MYH6, MYH7, troponin $T$ and connexin 43 were all at higher level in the cardiovascular construct when compared to the $\mathrm{CM}$ monoculture $(p<0.05)$ (Fig. 4).

In the cardiovascular construct, the expression levels of ion channels CACNAIC, KCNJ2 and SCN5A did not increase significantly between days 7-18 (Fig. 4). However, similarly to structural genes, there was statistically significant difference when expression levels were compared between cardiovascular construct and $\mathrm{CM}$ monoculture at day 18 . The 

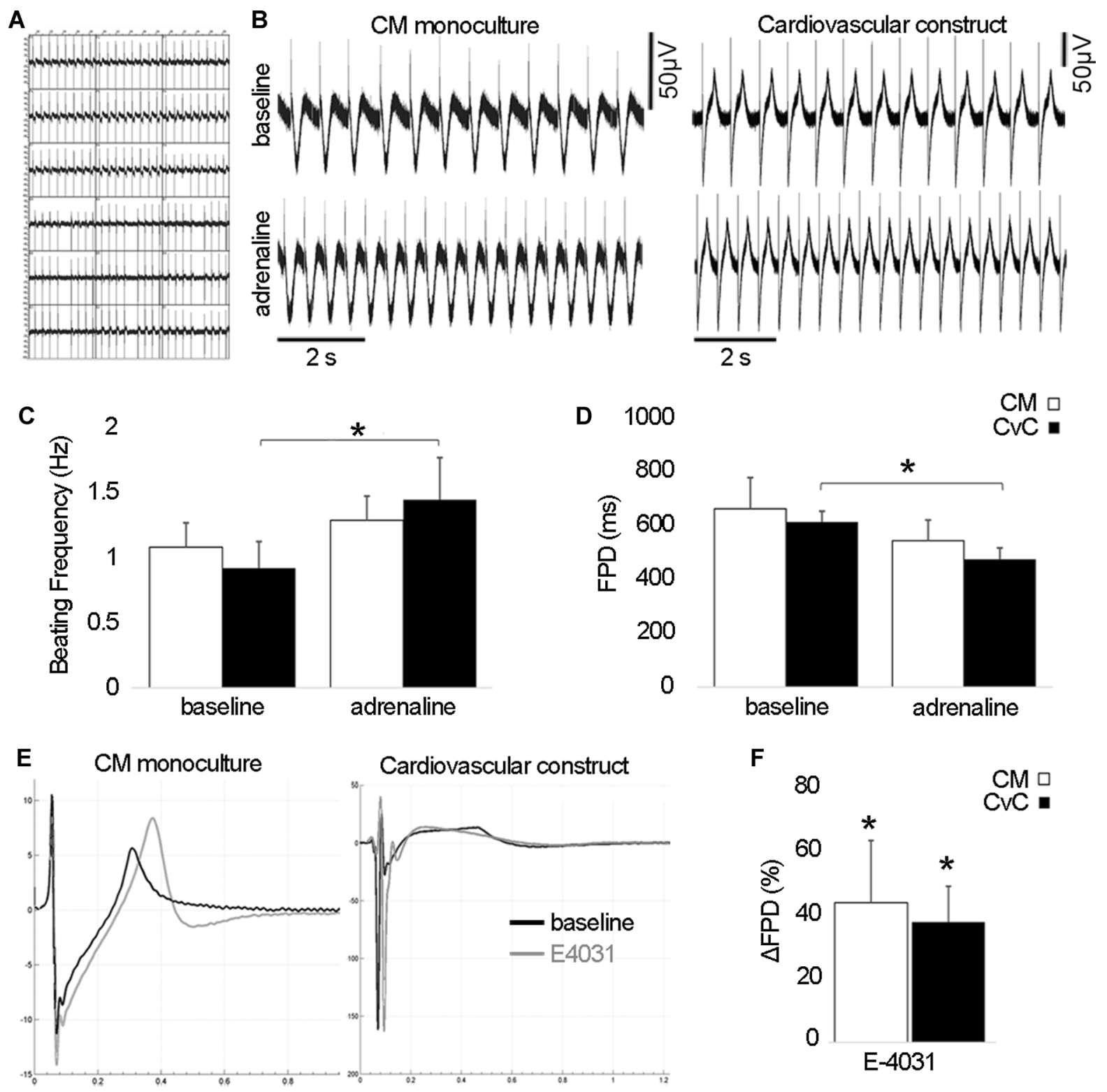

G

\begin{tabular}{|c|c|c|c|c|c|}
\cline { 2 - 6 } \multicolumn{1}{c|}{} & \multicolumn{3}{l}{$300 \mathrm{~nm}$ E-4031 } \\
\cline { 2 - 6 } \multicolumn{1}{c|}{} & $\mathrm{n}$ & Normal & $\begin{array}{c}\text { Normal + } \\
\text { Pause }\end{array}$ & Arrhythmia & $\begin{array}{c}\text { Arrhythmia + } \\
\text { Pause }\end{array}$ \\
\hline CM monoculture & 6 & 0 & 2 & 3 & 1 \\
Cardiovascular model & 7 & 2 & 1 & 3 & 1 \\
\hline
\end{tabular}

expression of beta-1 adrenergic receptor (ADRB1) was significantly higher at day 7 to day 18 in the cardiac construct $(p>0.05)$. Moreover, the expression was significantly higher at day 18 in the cardiovascular construct compared to $\mathrm{CM}$ monoculture. 
4Fig. 5 MEA measurements of the cardiovascular construct and CM monoculture. a The electrical activity of the cardiovascular construct was detectable with MEA and the contractility was synchronous; $\mathbf{b}$ representative field potential traces of the effect of $\beta$-adrenergic agonist adrenaline $(1 \mu \mathrm{M})$; $\mathbf{c}$ beating frequency and $\mathbf{d}$ field potential duration (FPD) of baseline and adrenaline measurements of CM monoculture and cardiovascular construct (both $\mathrm{n}=5$ ). e Overlay plot showing the effect of $300 \mathrm{nM}$ E-4031 on FPD; $f$ the effect of E-4031 on FPD was calculated as relative change from the baseline in both $\mathrm{CM}$ monoculture $(n=6)$ and cardiovascular construct $(n=7)$. In both of these FPD was significantly prolonged with E4031 when compared to baseline but there was no significant difference between $\mathrm{CM}$ monoculture and cardiovascular construct. $\mathrm{g}$ Adverse effects of E-4031 during the time of 2 min measurement. The number indicates how many replicates were affected. Normal: no arrhythmias, Normal + Pause: no arrhythmias but field potential activity terminates, Arrhythmia: arrhythmias affecting amplitude and frequency, Arrhythmia + Pause: arrhythmias leading to termination of beating activity. $C M$ cardiomyocyte monoculture, $C v C$ cardiovascular construct. $* p<0.05$

Electrophysiological changes in pluripotent stem cell derived cardiomyocytes in cardiovascular construct suggest functional improvement of the cardiomyocytes

Contractility areas of the cardiovascular model were large and synchronous and beating was stronger when compared to moderately beating CM monoculture with smaller separately beating areas (Video S1, S2 and S3). The electrical activity of the cardiovascular construct was detectable with MEA and the contractility was synchronous (Fig. 5a). Adrenaline increased the beating frequency of cardiomyocytes (Fig. 5b, c) thus decreasing the field potential duration (FPD) in cardiovascular constructs as well as in CM monoculture (Fig. 5d). The increase in beating frequency and decrease in FPD was statistically significant between baseline and adrenaline only in cardiovascular construct (Fig. 5c, $p<0.05$ ).

E4031, a hERG blocker, was shown to significantly prolong the FPD both in cardiovascular construct and in $\mathrm{CM}$ monoculture compared to the baseline (both $p<0.05$ ) but there was no significant difference between the groups (Fig. 5e, f). Prolongation of FPD due to E4031 exposure resulted in additional adverse effects including pausing beating and/or arrhythmias. These adverse effects were seen in CM monoculture as well as in cardiovascular construct (Fig. $5 \mathrm{~g}$ ).
$\mathrm{Ca}^{2+}$ transients of pluripotent stem cell derived cardiomyocytes in the absence and presence of vascular-like network

Cardiovascular construct and CM monoculture were shown to respond similarly to adrenaline exposure (See supplemental Fig. S2A). When compared to baseline, adrenaline caused a significant increase in beating frequency (Fig. 6d, $p<0.05$ ) and a decrease in peak duration in both systems (Fig. 6b) and, additionally, a significant decrease in amplitude in cardiovascular construct (Fig. 6c, $p<0.05$ ). The amplitude in cardiovascular construct was significantly lower at baseline as well as during adrenaline perfusion compared to those in CM monoculture (Fig. 6c, $p<0.05$ ).

The diastolic $\mathrm{Ca}^{2+}$ increased significantly in $\mathrm{CM}$ monoculture and in cardiovascular construct due to the adrenaline exposure (Fig. $6 \mathrm{a}, p<0.05$ ). $\mathrm{Ca}^{2+}$ transient changes were also analyzed separately for each cell as the ratio of adrenaline response divided by baseline response. The results showed that in cardiovascular construct adrenaline caused a significant increase in beating frequency and significant decrease in amplitude when compared to CM monoculture (see supplemental Fig. S2B, $p<0.05)$.

\section{Discussion}

The use of physiologically relevant in vitro model systems is a prerequisite for translating the preclinical data into clinical studies (Engle and Puppala 2013). In preclinical cardiotoxicity studies, the assessment of the risk for QT interval prolongation is part of the standard evaluation of new compounds as defined by the International Conference of Harmonization (ICH) Expert Working Group (Braam et al. 2010). Human pluripotent stem cell derived cardiomyocyte (hPSCCM) based test systems could improve the early detection of QT prolongation with the benefit of being of human origin (Pfannkuche et al. 2010a). However, it is widely acknowledged that hPSC-CMs possess fetal-like characteristics, e.g. contractility with proliferative capacity and embryonic-like electrophysiology. Although the factors affecting maturity remain largely unknown, cell line, culture time, culture type and other culture conditions appear to have effects on the maturity (Robertson et al. 2013). 

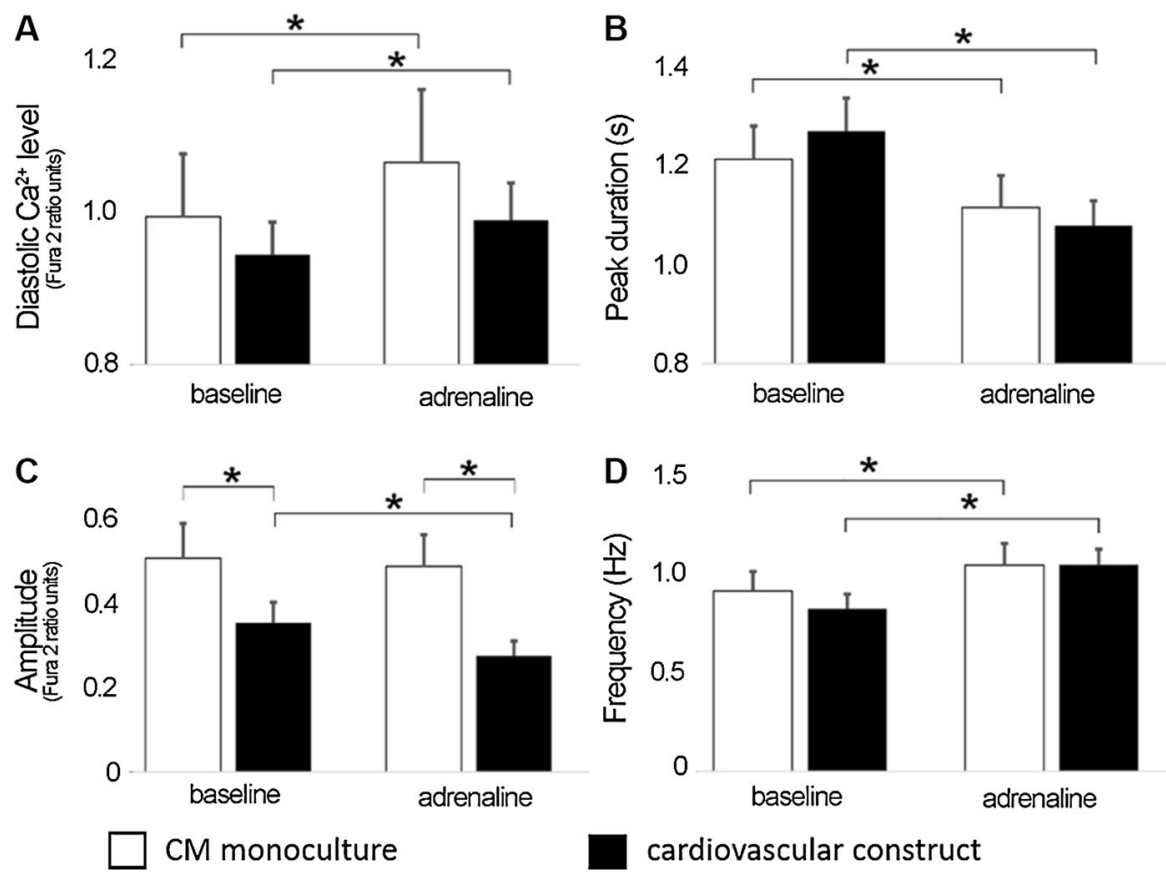

Fig. $6 \mathrm{Ca}^{2+}$ measurements of the cardiovascular construct (black columns) and cardiomyocyte monoculture (white columns) in baseline and after adrenaline exposure represented

We have previously developed cardiovascular constructs based on co-culture of neonatal rat cardiomyocytes and two different vascular-like networks formed either by human adipose stromal cells + HUVECs or HUVECs + fibroblasts. In the previous study, cardiomyocyte viability and functionality was maintained longer in co-cultures and morphological maturation was detected (Vuorenpaa et al. 2014). In the present study, vascular-like network, formed by HUVEC and fibroblasts, was combined with human cardiomyocytes to develop a completely human cell cardiovascular construct for cardiac safety and efficacy assessment and for biomedical research.

Expressions of cardiac structural protein coding genes MYH6 and MYH7, cardiac troponin T and gap junction marker connexin 43 were significantly increased in cardiovascular construct whereas they remained constant or decreased in CM monoculture. In CM monoculture, the overgrowth of non-cardiomyocytes may explain the decreased expression of structural transcripts MYH6, MYH7 and troponin $T$. However, similar overgrowth of non-cardiomyocytes was not detected in cardiovascular construct. Similar results were reported by Caspi et al. (2007) who

as a diastolic $\mathrm{Ca}^{2+}$ levels; b peak duration; $\mathbf{c}$ amplitude and d frequency. CM monoculture $\mathrm{n}=16$, cardiovascular construct $\mathrm{n}=26 . * p<0.05$

showed that genes coding structural proteins were expressed at higher level when cardiomyocytes were cultured with endothelial cells and fibroblasts in 3D biodegradable scaffold. Burridge et al. (2014) reported that troponin $T$ expression was significantly enhanced at protein level in the multicellular culture when compared to the CM monoculture. Our results are in line with the previous studies, suggesting that vascular-like network improves the cardiac specific phenotype of hPSC-CMs.

In heart, the extracellular matrix, mainly produced by the cardiac fibroblasts, guides cellular orientation and organization thus facilitating efficient cell contraction, force transduction and electrical transmission between the cells. Ventricular cardiomyocytes in the adult human heart are large and brick-shaped whereas hPSC-CMs are markedly smaller with round or triangular morphology (Hartman et al. 2015). The importance of alignment of cardiomyocytes in coordinated contraction is seen in the native cardiac structure, but is also shown in in vitro studies (van Spreeuwel et al. 2014). The alignment of cardiomyocytes has been induced in vitro by microcontact printing, demonstrating that alignment improves 
cardiomyocyte calcium handling and contractile properties when compared to randomly oriented cardiomyocyte monolayers (Pong et al. 2011; Feinberg et al. 2012). Our results demonstrate that seeding of hPSCCMs on already formed vascular-like network orientates the hPSC-CMs parallel with the tubule structures. Moreover, as detected in 3D projection, some hPSC$\mathrm{CMs}$ are seen to surround the tubule structures. Fibroblasts are known to produce extracellular matrix components and enhance alignment of cardiomyocytes (Pfannkuche et al. 2010b). The presence of fibroblasts is likely to enhance the orientation of hPSC-CMs with vascular-like structures.

Although earlier studies have shown that co-culture with endothelial cells and fibroblasts enhances the formation of more mature-like morphology of cardiomyocytes and increases the expression of cardiac specific genes and their electromechanical properties (Burridge et al. 2014; Radisic et al. 2008; Caspi et al. 2007), they are based on the utilization of different artificial scaffolds. Since the use of scaffolds in cardiac constructs has been associated with reduced cell-cell contacts, as well as incorrect deposition and alignment of extracellular matrix (Norotte et al. 2009), we aimed at inducing natural in vitro cardiovascular construct. We have previously shown that the vascular-like network formed by HUVEC and fibroblasts serves as a supporting platform for the rat cardiomyocytes (Vuorenpaa et al. 2014). In the present study, vascular-like network serves as a natural, supporting and interactive platform for the hPSC-CMs. Enhanced maturation of hPSC-CM detected already in 12 days suggests that the cardiovascular construct provides a more rapid test system. In addition, we detected increased sensitivity in response to adrenaline, which is likely due to higher expression of the $\beta 1$-adrenoreceptor in cardiovascular construct compared to $\mathrm{CM}$ monoculture.

Our current results with hPSC-CMs showed that the expression of several growth factors including $V E G F$, $F G F-2$, Ang-1, PDGF- $\beta$ and $T G F-\beta$ was active in vascular-like network throughout the experiment. In cardiovascular construct, expression levels of growth factors had a similar trend as in the vascular-like network, except for $V E G F$ and $P D G F-\beta$ that were increased in the cardiovascular construct. In vascular network, when endothelial cells assemble into tubular structures, endothelial cells start expressing $P D G F-B$ to recruit $P D G F-B$ receptor positive pericytes to surround the endothelial structures, a step required for tubular maturation. Endothelial cells also secrete $P D G F-B$ and other factors in order to modulate cardiomyocytes and assist in the development of heart tissue. As VEGF is mainly produced by the cardiomyocytes (Brutsaert 2003; Leucker et al. 2011), the increase in the expression level of $V E G F$ at day 18 is in line with the time in culture. In addition, Ang-2, a marker of early stage tubule formation that is expressed when sprouting of tubules and formation of new tubules occurs, was shown to increase in vascular-like network suggesting ongoing tubule formation process in vascular-like network. However, Ang-2 was low in cardiovascular construct, indicating more mature vascular structures, a result that is also in line with the increased $P D G F-\beta$ expression during culture. Therefore, cardiovascular construct, deprived of exogenous growth factors, was capable of activating the growth factor expression and maintain the differentiation process endogenously.

Our current results suggest that mature vascularlike structures seem not to be the prerequisite for the development of functional cardiovascular construct. More important seems to be the interactive natural stromal support that endothelial cells and fibroblasts provide to the construct. These cells induce cardiomyocytes by secreting several growth factors (Brutsaert 2003; Leucker et al. 2011; Pfannkuche et al. 2010b; Wong et al. 2007) and by providing e.g. endothelialcardiomyocyte contacts, known to be crucial in maintaining the rhythmic and synchronous contraction of cardiomyocytes (Brutsaert 2003). These cell-cell and cell-matrix interactions as well as paracrine signals of the co-cultured cells, seemed to be critical for the viability of hPSC-CMs and functionality of the developed cardiovascular construct. Although the vascular-like network formation and differentiation cease in the absence of VEGF and FGF-2 during hPSC-CM culture, the cardiomyocyte viability or contractility, as detected in the MEA and calcium metabolism measurements, are enhanced in the presence of the network.

Although the major ionic currents normally present in adult cardiomyocytes are expressed also in hPSCCMs (Robertson et al. 2013), differences are seen in the expression levels of cardiac ion channels and in calcium handling genes (Synnergren et al. 2007). Our gene expression data showed that in the presence of vascular-like network, the expression of transcripts of 
sodium (SCN5A) and calcium (CACNA1C) channels were increased during the days 7-18, while the level of potassium channel $(K C N J 2)$ remained constant. More importantly, all these genes were expressed at higher level in the cardiovascular construct compared to CM monoculture at the end of the experiment (day 18). $S C N 5 A$ gene codes the cardiac sodium channel $\mathrm{Na}_{\mathrm{V}} 1.5$ which functions in the fast depolarization phase of the cardiac action potential. The expression level of SCN5A has been reported to increase when hPSCCMs were maturated upon electrical stimulation (Chan et al. 2013). In addition, the expression of CACNA1C, gene coding the L-type $\mathrm{Ca}^{+}$-channel Cav 1.2, was shown to increase in co-culture with hESCderived endothelial cells and human amniotic mesenchymal stem cells (Burridge et al. 2014). The expression level of KCNJ2 that codes the Kir2.1 channel responsible for the inward rectifier potassium current $\left(\mathrm{I}_{\mathrm{K} 1}\right)$, has been reported to significantly increase during hESC-CM maturation (Sartiani et al. 2007) and in culture with endothelial cells (Burridge et al. 2014). Despite the relative short co-culture time with vascular-like network, cardiomyocytes in cardiovascular construct expressed these ion channelcoding genes at higher level compared to CM monoculture. The increased expression indicates that the aforementioned currents are increasingly present in the cardiovascular construct and the hPSC-CMs would therefore exhibit electrophysiologically more mature phenotype than the monoculture of cardiomyocytes.

MEA measurements showed that E-4031 increased significantly the field potential duration and arrhythmogenicity in the cardiovascular construct as well as in $\mathrm{CM}$ monoculture. Calcium imaging analysis showed that $\mathrm{Ca}^{2+}$ transients were detectable and adrenaline responses evident in the cardiovascular construct. We detected that adrenaline increased significantly the beating frequency of cardiovascular construct compared to $\mathrm{CM}$ monoculture. This was evident in field potential recordings as well as in $\mathrm{Ca}^{2+}$ cycling measurements and further confirmed by gene expression analysis. A significantly higher expression of $A D R B 1$, a gene encoding the $\beta 1$-adrenoreceptor, was detected in the cardiovascular construct compared to $\mathrm{CM}$ monoculture which further supports the conclusion that vascular-like network has positive effect on the maturation status of the hPSC-CMs.

The specific mechanism how vascular-like network enhances the maturation state of hPSC-CMs remains unknown. Comparative analysis between cardiovascular construct and adult human myocardium could give more detailed information about the maturation state of the construct. However, it can be hypothesized that neighboring non-myocytes could enhance the electrical maturation of hPSC-CMs in cardiovascular construct by secreting paracrine factors or non-cardiac cells might enhance cardiomyocyte signal propagation through cell-to-cell contacts as also suggested by others (Sekine et al. 2008; Narmoneva et al. 2004). Our results suggest that cardiovascular construct presents a more mature in vitro cardiac model compared to $\mathrm{CM}$ monoculture and could therefore serve as an advanced test system for cardiac safety and efficacy assessment as well as a model system for biomedical research.

Acknowledgements We sincerely thank Ms. Mirja Hyppönen, Ms. Paula Helpiölä, Ms. Sari Leinonen, Ms. Hilkka Mäkinen and Ms. Henna Venäläinen for the excellent technical assistance. We also want to thank Dr. Erja Kerkelä for her contribution to this work. This study was supported by the TEKES-Finnish Funding Agency for Innovation, Ministry of Education and Culture, Ministry of Agriculture and Forestry and Finnish Cardiovascular Foundation. In addition, FinPharma Doctoral Programme, Orion Pharma, Pirkanmaa Hospital District Foundation and Finnish Cultural Foundation supported this work.

\section{Compliance with ethical standards}

Conflict of interest None declared.

Open Access This article is distributed under the terms of the Creative Commons Attribution 4.0 International License (http:// creativecommons.org/licenses/by/4.0/), which permits unrestricted use, distribution, and reproduction in any medium, provided you give appropriate credit to the original author(s) and the source, provide a link to the Creative Commons license, and indicate if changes were made.

\section{References}

Bhattacharya S, Macdonald ST, Farthing CR (2006) Molecular mechanisms controlling the coupled development of myocardium and coronary vasculature. Clin Sci (London, England: 1979) 111:35-46

Braam SR, Tertoolen L, van de Stolpe A, Meyer T, Passier R, Mummery CL (2010) Prediction of drug-induced cardiotoxicity using human embryonic stem cell-derived cardiomyocytes. Stem Cell Res 4:107-116

Brutsaert DL (2003) Cardiac endothelial-myocardial signaling: its role in cardiac growth, contractile performance, and rhythmicity. Physiol Rev 83:59-115 
Burridge PW, Metzler SA, Nakayama KH, Abilez OJ, Simmons CS, Bruce MA, Matsuura Y, Kim P, Wu JC, Butte M, Huang NF, Yang PC (2014) Multi-cellular interactions sustain long-term contractility of human pluripotent stem cell-derived cardiomyocytes. Am J Transl Res 6:724-735

Caspi O, Lesman A, Basevitch Y, Gepstein A, Arbel G, Habib IH, Gepstein L, Levenberg S (2007) Tissue engineering of vascularized cardiac muscle from human embryonic stem cells. Circ Res 100:263-272

Chan YC, Ting S, Lee YK, Ng KM, Zhang J, Chen Z, Siu CW, Oh SK, Tse HF (2013) Electrical stimulation promotes maturation of cardiomyocytes derived from human embryonic stem cells. J Cardiovasc Transl Res 6:989-999

Chien KR, Domian IJ, Parker KK (2008) Cardiogenesis and the complex biology of regenerative cardiovascular medicine. Science (New York, NY) 322:1494-1497

Davila JC, Cezar GG, Thiede M, Strom S, Miki T, Trosko J (2004) Use and application of stem cells in toxicology. Toxicol Sci Off J Soc Toxicol 79:214-223

Engle SJ, Puppala D (2013) Integrating human pluripotent stem cells into drug development. Cell Stem Cell 12:669-677

Feinberg AW, Alford PW, Jin H, Ripplinger CM, Werdich AA, Sheehy SP, Grosberg A, Parker KK (2012) Controlling the contractile strength of engineered cardiac muscle by hierarchal tissue architecture. Biomaterials 33:5732-5741

Feric NT, Radisic M (2016) Maturing human pluripotent stem cell-derived cardiomyocytes in human engineered cardiac tissues. Adv Drug Deliv Rev 96:110-134

Garzoni LR, Rossi MID, de Barros APDN, Guarani V, Keramidas M, Balottin LBL, Adesse D, Takiya CM, Manso PP, Otazú IB, Meirelles Mde N, Borojevic R (2009) Dissecting coronary angiogenesis: $3 \mathrm{D}$ co-culture of cardiomyocytes with endothelial or mesenchymal cells. Exp Cell Res 315:3406-3418

Hartman ME, Dai DF, Laflamme MA (2015) Human pluripotent stem cells: prospects and challenges as a source of cardiomyocytes for in vitro modeling and cell-based cardiac repair. Adv Drug Deliv Rev 96:3-17

Hsieh PC, Davis ME, Lisowski LK, Lee RT (2006) Endothelialcardiomyocyte interactions in cardiac development and repair. Annu Rev Physiol 68:51-66

Kettenhofen R, Bohlen H (2008) Preclinical assessment of cardiac toxicity. Drug Discov Today 13:702-707

Lahti AL, Kujala VJ, Chapman H, Koivisto AP, PekkanenMattila M, Kerkela E, Hyttinen J, Kontula K, Swan H, Conklin BR, Yamanaka S, Silvennoinen O, Aalto-Setälä K (2012) Model for long QT syndrome type 2 using human iPS cells demonstrates arrhythmogenic characteristics in cell culture. Dis Models Mech 5:220-230

Leucker TM, Bienengraeber M, Muravyeva M, Baotic I, Weihrauch D, Brzezinska AK, Warltier DC, Kersten JR, Pratt PF Jr. (2011) Endothelial-cardiomyocyte crosstalk enhances pharmacological cardioprotection. J Mol Cell Cardiol 51:803-811

Lian X, Zhang J, Azarin SM, Zhu K, Hazeltine LB, Bao X, Hsiao C, Kamp TJ, Palecek SP (2013) Directed cardiomyocyte differentiation from human pluripotent stem cells by modulating Wnt/beta-catenin signaling under fully defined conditions. Nat Protoc 8:162-175

Livak KJ, Schmittgen TD (2001) Analysis of relative gene expression data using real-time quantitative PCR and the 2(-delta delta C(T)) method. Methods (San Diego, Calif) 25:402-408

Martin RL, McDermott JS, Salmen HJ, Palmatier J, Cox BF, Gintant GA (2004) The utility of hERG and repolarization assays in evaluating delayed cardiac repolarization: influence of multi-channel block. J Cardiovasc Pharmacol 43:369-379

Mummery C, Ward-van Oostwaard D, Doevendans P, Spijker R, van den Brink S, Hassink R, van der Heyden M, Opthof T, Pera M, de la Riviere AB, Passier R, Tertoolen L (2003) Differentiation of human embryonic stem cells to cardiomyocytes: role of coculture with visceral endoderm-like cells. Circulation 107:2733-2740

Narmoneva DA, Vukmirovic R, Davis ME, Kamm RD, Lee RT (2004) Endothelial cells promote cardiac myocyte survival and spatial reorganization: implications for cardiac regeneration. Circulation 110:962-968

Norotte C, Marga FS, Niklason LE, Forgacs G (2009) Scaffoldfree vascular tissue engineering using bioprinting. Biomaterials 30:5910-5917

Pfannkuche K, Hannes T, Khalil M, Noghabi MS, Morshedi A, Hescheler J, Droge P (2010a) Induced pluripotent stem cells: a new approach for physiological research. Cell Physiol Biochem Int J Exp Cell Physiol Biochem Pharmacol 26:105-124

Pfannkuche K, Neuss S, Pillekamp F, Frenzel LP, Attia W, Hannes T, Salber J, Hoss M, Zenke M, Fleischmann BK, Hescheler J, Sarić T (2010b) Fibroblasts facilitate the engraftment of embryonic stem cell-derived cardiomyocytes on three-dimensional collagen matrices and aggregation in hanging drops. Stem Cells Dev 19:15891599

Polini A, Prodanov L, Bhise NS, Manoharan V, Dokmeci MR, Khademhosseini A (2014) Organs-on-a-chip: a new tool for drug discovery. Expert Opin Drug Discov 9:335-352

Pong T, Adams WJ, Bray MA, Feinberg AW, Sheehy SP, Werdich AA, Parker KK (2011) Hierarchical architecture influences calcium dynamics in engineered cardiac muscle. Exp Biol Med (Maywood, NJ) 236:366-373

Pontes Soares C, Midlej V, de Oliveira ME, Benchimol M, Costa ML, Mermelstein C (2012) 2D and 3D-organized cardiac cells shows differences in cellular morphology, adhesion junctions, presence of myofibrils and protein expression. PLoS ONE 7:e38147

Pradhapan P, Kuusela J, Viik J, Aalto-Setala K, Hyttinen J (2013) Cardiomyocyte MEA data analysis (CardioMDA) - a novel field potential data analysis software for pluripotent stem cell derived cardiomyocytes. PLoS One 8:e73637

Radisic M, Park H, Martens TP, Salazar-Lazaro JE, Geng W, Wang Y, Langer R, Freed LE, Vunjak-Novakovic G (2008) Pre-treatment of synthetic elastomeric scaffolds by cardiac fibroblasts improves engineered heart tissue. J Biomed Mater Res Part A 86:713-724

Robertson C, Tran DD, George SC (2013) Concise review: maturation phases of human pluripotent stem cell-derived cardiomyocytes. Stem Cells (Dayton, Ohio) 31:829-837

Sarkanen JR, Mannerstrom M, Vuorenpaa H, Uotila J, Ylikomi $\mathrm{T}$, Heinonen T (2011) Intra-laboratory pre-validation of a human cell based in vitro angiogenesis assay for testing angiogenesis modulators. Front Pharmacol 1:147 
Sartiani L, Bettiol E, Stillitano F, Mugelli A, Cerbai E, Jaconi ME (2007) Developmental changes in cardiomyocytes differentiated from human embryonic stem cells: a molecular and electrophysiological approach. Stem Cells (Dayton, Ohio) 25:1136-1144

Schaaf S, Shibamiya A, Mewe M, Eder A, Stohr A, Hirt MN, Rau T, Zimmermann WH, Conradi L, Eschenhagen T, Hansen A (2011) Human engineered heart tissue as a versatile tool in basic research and preclinical toxicology. PLoS ONE 6:e26397

Sekine H, Shimizu T, Hobo K, Sekiya S, Yang J, Yamato M, Kurosawa H, Kobayashi E, Okano T (2008) Endothelial cell coculture within tissue-engineered cardiomyocyte sheets enhances neovascularization and improves cardiac function of ischemic hearts. Circulation 118:S145-S152

Snir M, Kehat I, Gepstein A, Coleman R, Itskovitz-Eldor J, Livne E, Gepstein L (2003) Assessment of the ultrastructural and proliferative properties of human embryonic stem cell-derived cardiomyocytes. Am J Physiol Heart Circ Physiol 285:H2355-H2363

Soonpaa MH, Field LJ (1998) Survey of studies examining mammalian cardiomyocyte DNA synthesis. Circ Res $83: 15-26$

Synnergren J, Giesler TL, Adak S, Tandon R, Noaksson K, Lindahl A, Nilsson P, Nelson D, Olsson B, Englund MC, Abbot S, Sartipy P (2007) Differentiating human embryonic stem cells express a unique housekeeping gene signature. Stem Cells 25:473-480

Takahashi K, Tanabe K, Ohnuki M, Narita M, Ichisaka T, Tomoda K, Yamanaka S (2007) Induction of pluripotent stem cells from adult human fibroblasts by defined factors. Cell 131:861-872

Valarmathi MT, Goodwin RL, Fuseler JW, Davis JM, Yost MJ, Potts JD (2010) A 3-D cardiac muscle construct for exploring adult marrow stem cell based myocardial regeneration. Biomaterials 31:3185-3200

van Spreeuwel AC, Bax NA, Bastiaens AJ, Foolen J, Loerakker S, Borochin M, van der Schaft DW, Chen CS, Baaijens FP, Bouten CV (2014) The influence of matrix (an)isotropy on cardiomyocyte contraction in engineered cardiac microtissues. Integr Biol Quant Biosci Nano Macro 6:422-429

Vuorenpaa H, Ikonen L, Kujala K, Huttala O, Sarkanen JR, Ylikomi T, Aalto-Setala K, Heinonen T (2014) Novel in vitro cardiovascular constructs composed of vascularlike networks and cardiomyocytes. In Vitro Cell Dev Biol Anim 50:275-286

Wong T, McGrath JA, Navsaria H (2007) The role of fibroblasts in tissue engineering and regeneration. $\mathrm{Br} \mathrm{J}$ Dermatol 156:1149-1155

Yang X, Pabon L, Murry CE (2014) Engineering adolescence: maturation of human pluripotent stem cell-derived cardiomyocytes. Circ Res 114:511-523 